doi: $10.35366 / 100003$

\title{
Humanización en la Unidad de Cuidados Intensivos
}

Humanization in Intensive Care Unit

Humanização em Unidade de Terapia Intensiva

Ricardo Martínez Zubieta*

\section{RESUMEN}

La humanización en la Unidad de Cuidados Intensivos es una necesidad actual ante la evidencia de mejores resultados en la evolución de los enfermos, mayor satisfacción en la familia y apoyo al personal de la salud que labora dentro de las mismas. Mucha de dicha evidencia se presenta en aquellas unidades de puertas abiertas, donde el enfermo se siente más acompañado de quien é decide, la familia participa más en su cuidado y sobre todo se fomenta a través de una mayor comunicación la autonomía de este binomio.

Palabras clave: Humanización, Unidad de Cuidados Intensivos, cuidados intensivos, cuidados centrados en el enfermo y familia.

\section{ABSTRACT}

Humanization in the Intensive Care Unit is a current need in the face of evidence of better results in the evolution of the patients. It also brings greater satisfaction of the family members and provides support for the health personnel working within them. Much of this evidence is presented in those open-door units, where the patient feels more accompanied by whom he decides, the family members participate more in the care of the patient and, above all, the autonomy of this binomial is promoted through greater communication.

Keywords: Humanization, Intensive Care Unit, intensive care, patient and family centered-care.

\section{RESUMO}

A humanização na Unidade de Terapia Intensiva é uma necessidade atual diante das evidências de melhores resultados na evolução dos pacientes, maio satisfação na família e apoio aos profissionais de saúde que nela atuam. Muitas dessas evidências são apresentadas nessas unidades de portas abertas, onde o paciente se sente mais acompanhado por quem decide, a família participa mais do seu cuidado e, sobretudo, a autonomia desse binômio é promovida por meio de uma maior comunicação.

Palavras-chave: Humanização, Unidade de Cuidados Intensivos, cuidados intensivos, cuidado centrado no paciente e na família.

\section{INTRODUCCIÓN}

Es frecuente que demandemos un mejor servicio al que tenemos derecho, diciéndole a la persona frente a nosotros que el servicio que nos están proporcionando es muy deficiente, incluyendo una conducta displicente. También todos los días que hablamos por teléfono a un banco escuchamos una grabación que por lo general nos da más de dos opciones, después de escoger una, siguen otras más, y así seguimos de una opción a otra, y quizá con suerte nos responda una persona muy amable, pero a quien no vemos su cara, no podemos determinar su comunicación no verbal (actitud), y quizá aún con sus respuestas quedamos insatisfechos por éstas. Finalmente dicha persona nos pide que respondamos a una encuesta de satisfacción.

\footnotetext{
* Hospital Español de México. Grupo Hospital Español.

Recibido: 26/02/2020. Aceptado: 30/03/2020

Citar como: Martínez ZR. Humanización en la Unidad de Cuidados Intensivos. Med Crit. 2021;35(3):144-147. https://dx.doi.org/10.35366/100003

www.medigraphic.com/medicinacritica
}

Todo esto ahora lo llevo a nuestra bella actividad, donde con frecuencia tenemos una conducta muy parecida a lo descrito en las primeras líneas de este artículo. Ejemplos de dicha actitud son: 1) «ya no es hora de visita» (el visitante es la madre o padre, hermana, esposo, hijo, etcétera, quien sólo puede visitar a su enfermo en un horario no compatible por cuestiones personales); 2) «yo no sé nada de su enfermo, pregúntele a su médico»; 3) «isálgase del cuarto!», o mejor, «¿se puede usted salir del cuarto? Ya que voy a darle un medicamento»; 4) "su enfermo tiene 'un SIRA' y para eso requiere una ' $\mathrm{FiO}_{2}$ ' y un 'PEEP' elevados, además de que iniciaremos una terapia de reemplazo renal continua»; 5) «su enfermo puede morir como lo predicen las escalas de gravedad como SOFA, APACHE 2 y Glasgow muy altas»; y 6) otras muchas.

¿Qué diferencia existe entre las situaciones que vivimos como demandantes y en la que somos los demandados?, quizá no mucha. De inicio no parece que existan diferencias, pero sí existen y puedo asegurar que estamos en desventaja si somos los demandados, ya que nosotros trabajamos con la salud y el dolor, con esperanzas o desesperanzas, con cargas emocionales, familiares y ahora, muy frecuente, económicas.

Esta conducta puede llamarse «deshumanización en la Unidad de Cuidados Intensivos (UCls)», no es infrecuente y somos responsables médicos, enfermeras, técnicos, personal administrativo y directivo de los hospitales.

Ante este comportamiento humano (o deshumano) del equipo de salud, y con un sentido de alta responsabilidad en nuestro actuar, ya se han publicado guías y recomedanciones ${ }^{1,2}$ que destacan la necesidad de trabajar más en un cuidado centrado en el enfermo y su familia, mejorando nuestra conducta y falta de sensibilidad. Asimismo, ya existen iniciativas en otros países, por ejemplo, en Estados Unidos de América por la Sociedad Americana de Medicina Crítica, ${ }^{1}$ y en España, por iniciativa del Dr. Gabriel Heras, han desarrollado el proyecto «Humanizando los Cuidados Intensivos: Proyecto HU-Cl» (www. humanizandoloscuidadosintensivos.com/es).

En la actualidad, han lanzado un Manual de Buenas Prácticas de Humanización de las Unidades de Cuidados Intensivos para favorecer un entorno más amable para enfermos, familiares y profesionales de la salud. Dicho proyecto tiene varios objetivos dirigidos a lograr mayor beneficio en los enfermos, familiares y equipo de salud, pero resalto con diversa bibliografía algunos de ellos sin dejar de reconocer la importancia de los 
restantes: $\mathrm{UCI}$ de puertas abiertas, cuidados centrados en el enfermo, su familia y el personal de salud, mayor participación de los familiares en el cuidado del enfermo y finalmente mejor comunicación. ${ }^{3-7}$

Los otros objetivos de este programa son: cuidados al final de la vida, infraestructura humanizada, cuidados al personal, prevención, atención y seguimiento del síndrome postcuidados intensivos (SPCI). ${ }^{4}$

\section{$\mathrm{UCl}$ de puertas abiertas}

En la literatura médica se pueden encontrar muchos trabajos que han demostrado el beneficio de UCls de puertas abiertas, mayor satisfacción del enfermo (dependiendo de la patología de base) y de sus familiares, así como de los propios trabajadores de la salud. En su reflexión Magdalena Llesuy ${ }^{7}$ destaca que el permitir que los enfermos permanezcan el mayor tiempo posible acompañados por quien ellos decidan y que «las normas y los protocolos deben diseñarse y rediseñarse, haciéndolos más flexibles y adaptables a las necesidades reales de los enfermos (y agrego yo: también a los familiares) garantizan su máximo bienestar y beneficio». En ningún caso se debe permitir que el enfermo se convierta en una víctima pasiva de las normas y de los protocolos. Por ejemplo, es frecuente que algún acompañante no coincida con los horarios establecidos en la $\mathrm{UCI}$ por diversos motivos (personales y/o laborales), y esta condición no debe impedir el acceder a la compañía en un horario determinado, por ejemplo nocturno.

¿Pero cómo definir una $\mathrm{UCI}$ de puertas abiertas? Creo que la descripción que hace el pediatra italiano Alberto Giannini es bastante completa y correcta; los objetivos de la UCl abierta son reducir o eliminar toda limitación impuesta en tres dimensiones: temporal, física (contacto) y de relación (comunicación poco clara, incompleta o inexistente) en la que no exista justificación. ${ }^{8}$ El mismo autor recomienda tener como requisito de acreditación hospitalaria en el Servicio Nacional de Salud de Italia las visitas no restringidas en las UCls. ${ }^{8}$

\section{Efectos benéficos en los enfermos}

Las ventajas en los enfermos se han descrito en varios aspectos, mentales, fisiológicos y en la evaluación de la satisfacción. Aliberch y Miquel describen una disminución del estrés y mayor satisfacción tanto en los enfermos como en los familiares cuando éste es acompañado por un familiar. ${ }^{9}$ El manejo del dolor, síntoma que puede ser mejor percibido por la continua presencia de familiares, puede ser optimizado (sobre todo en niños). ${ }^{10}$

Otros autores han reportado mayor satisfacción del enfermo cuando sus familiares participan en su cuidado, ${ }^{11}$ mientras que Romero García y colaboradores describen como satisfactorio el cuidado por enfermería que se caracteriza por un abordaje humanístico y científico. ${ }^{12}$ Fumagalli y su grupo demostraron en un estudio aleatorizado de visitas restringidas versus visitas no restringidas de enfermos en la $\mathrm{UCl}$ los siguientes datos: a) similar presencia de complicaciones sépticas; b) un riesgo estadísticamente significante de complicaciones cardiovasculares en enfermos con visitas restringidas, (OR 2.0; IC 95\%, 1.1 a 3.5; $p=0.03$ ); c) una similar mortalidad (5.2 versus $1.8 ; p=0.28)$; $d$ ) mayor reducción en el índice de ansiedad; y e) menor incremento de la hormona estimulante de la tiroides desde el momento de admisión al alta de la $\mathrm{UCl} .{ }^{13}$ Otro aspecto también investigado es el desarrollo de delirio en enfermos durante su estancia en la $\mathrm{UCl}$, y este se ha reportado que disminuye de forma importante en enfermos ingresados en unidades sin restricción en el acompañamiento. ${ }^{14,15}$

De manera reciente, se han descrito otros problemas emocionales y cognitivos agudos y crónicos como ansiedad, estrés, depresión y el desarrollo del síndrome postcuidados intensivos (SPCI) en enfermos que permanecen sin compañía. ${ }^{16,17}$ Wolters y colegas encontraron en una encuesta realizada a tres meses después del alta hospitalaria a enfermos ingresados en la $\mathrm{UCI}$ una marcada presencia de alteraciones psicológicas, con $74 \%$ presentando depresión, 59\% ansiedad y $15 \%$ $\mathrm{SPCl} .{ }^{18}$ De forma paralela, este último síndrome también se encontró en $15 \%$ de los miembros de la familia del enfermo. ${ }^{18}$

En resumen, muchas alteraciones fisiológicas, mentales y emocionales en los enfermos pueden desarrollarse por condiciones usualmente presentes en la $\mathrm{UCI}$ o presentes en los enfermos; en los primeros encontramos aislamiento, medio ambiente muy tecnificado que nos guía en la atención de los enfermos y nos hace olvidar al enfermo humano, limitada comunicación entre el enfermo y el equipo de la $\mathrm{UCl}$, así como un lenguaje muy técnico, movimiento limitado o incomodidad del enfermo, exceso de luz y ruido que condicionan alteraciones del sueño, uso de restricciones físicas (sujeciones), invasión física con dispositivos necesarios en el tratamiento (catéteres y sondas, ventiladores mecánicos, equipos de sustitución renal y otro tipo de monitores); otros factores son propios del enfermo como sed, dolor y miedo. Por último, la pérdida de su identidad y autonomía en la toma de decisiones importantes de modo frecuente está presente, incluso para sus acompañantes.

Por otra parte, la decisión de la compañía del enfermo debe ser determinada por él mismo. En otras palabras, él decide si desea un acompañante (que en algunas ocasiones no es un familiar directo, puede ser una amistad quien conoce más sus preferencias, deseos y miedos). Holanda Peña y su equipo encontraron en un estudio de satisfacción poca concordancia entre lo opinado por los enfermos y lo opinado por sus familiares, concluyendo que estos últimos pueden no ser siempre 
considerados los representantes más adecuados (al menos de enfermos competentes). ${ }^{19}$

Harvey y colaboradores, ya desde 1993 en la descripción de autonomía del enfermo, detallaron aspectos muy interesantes inherentes a este término: 1) conocer las necesidades, valores, espiritualidad (que describen como la «vida interna del enfermo» y que no necesariamente se limita a la religión); 2) decidir la compañía y quién es esa compañía; 3) fomentar la participación del enfermo en su recuperación, esto bajo la vigilancia de personal capacitado; 4) tener flexibilidad en el plan de cuidado del enfermo cuando sus deseos entren en conflicto con el plan establecido; y 5) ofrecer siempre consentimiento informado con derecho de rechazo, a limitación de esfuerzos terapéuticos e incluso orden de no reanimación. ${ }^{20}$

\section{Efectos benéficos en las familias}

Algunos autores han descrito que la visita en las UCls ha sido tradicionalmente prohibida o restringida con la creencia de que los acompañantes son una amenaza a los enfermos por aumento en el riesgo de infecciones y mayor estrés, así como un obstáculo en los cuidados del enfermo. Todo lo contrario se ha observado en las UCls de puertas abiertas, habiéndose descrito diversas e importantes ventajas: 1) trabajar en cooperación, con la atenta vigilancia de los familiares de los enfermos; 2) proporcionar tranquilidad y seguridad de que su ser querido está siendo atendido de forma continua; 3) facilitar la comunicación; y 4) aumentar la confianza. 8,21

Rodríguez Martínez y colegas encontraron que una mayor participación de los familiares en el cuidado del paciente crítico se acompañó de mayor satisfacción de la familia $(p=0.026)$, mayor apoyo emocional al enfermo $(p=0.05)$, mayor percepción del dolor $(p=0.013)$ y como consecuencia una disminución significativa del grado de ansiedad y mayor aceptación de las enfermeras con respecto a una vista abierta. ${ }^{22}$ Similares resultados reportaron Garrouste-Orgeas y su grupo al reportar que los enfermos (77.2\%) y el equipo de salud percibieron de manera favorable la participación de los familiares en algunas actividades seleccionadas del cuidado del enfermo en la UCI. ${ }^{11}$

\section{Efecto benéfico en los profesionales de la salud}

Un mayor reconocimiento del trabajo y mejores relaciones humanas con el equipo de salud de la $\mathrm{UCl}$ son algunos de los beneficios que ha mostrado la $\mathrm{UCI}$ de puertas abiertas, destacando los autores la participación del personal de enfermería en la información al enfermo y/o familia. ${ }^{23}$ Este hecho indudablemente reconoce a dicho personal en el trabajo conjunto dentro de las UCIs.
Jover y colegas (al igual que Romero y su equipo ${ }^{12}$ ) demostraron en su estudio que la percepción de los enfermos en la $\mathrm{UCl}$ es satisfactoria cuando los cuidados de enfermería se proporcionan de forma integral, continua y segura, logrando bienestar y confianza, y dichas percepciones también fueron captadas por el personal de enfermería. ${ }^{24}$

Por otra parte, la necesidad de humanizar las UCls ha sido destacada en otros trabajos, al modificar el modelo de atención a través de mejorar la comunicación, el cuidado con mayor respeto del enfermo, un mayor acercamiento y soporte a la familia y al favorecer un trabajo multidisciplinario. ${ }^{25,26}$

\section{CONCLUSIÓN}

La humanización de las UCls y de todo servicio de salud en la actualidad debe ser obligada. La evidencia actual demuestra los grandes beneficios en los enfermos, familiares y equipo de salud. El dar la espalda a esta iniciativa ya de muchos países favorece la deshumanización; esta es ya endémica como ha sido descrita en la literatura, teniendo muchas causas, algunas muy complejas, pero que tienen probables soluciones (recomiendo mucho la lectura de este artículo). ${ }^{27}$

\section{REFERENCIAS}

1. Davidson JE, Aslakson RA, Long AC, Puntillo KA, Kross EK, Hart $\mathrm{J}$, et al. Guidelines for Family-Centered Care in the Neonatal, Pediatric, and Adult ICU. Crit Care Med. 2017;45:103-128.

2. Davidson JE. Family-Centered Care: Meeting the Needs of Patients' Families and Helping Families Adapt to Critical IIIness. Critical Care Nurse. 2009;29:28-34.

3. Alonso OA, Heras LG. ICU: a branch of hell? Intensive Care Med. 2016;42:591-592.

4. Heras LG, Alonso OA, Gómez TV. A plan for improving the humanisation of intensive care units. Intensive Care Med. 2017; $43: 547-549$

5. Curtis J, Sprung C, Azoulay E. The importance of word choice in the care of critically ill patients and their families. Intensive Care Med. 2014;40:606-608.

6. Canabal CA, Hernández MG. ¿Puede la satisfacción de los pacientes y familiares influir en la gestión de los servicios de medicina intensiva? Med Intensiva. 2017;41:67-69.

7. Llesuy SM. Algunas reflexiones a propósito de las visitas en las unidades de cuidados intensivos. Enferm Intensiva. 2002;13:17-19.

8. Giannini A. The "open" ICU: not just a question of time. Minerva Anestesiol. 2010;76:89-90.

9. Aliberch RAM, Miquel AIM. Necesidad de rol en los familiares del paciente en la unidad de cuidados intensivos. Enferm Intensiva. 2015;26:101-111.

10. Rodríguez MC, Rodríguez F, Roncero A, Morgado MI, Theodor J, Flores CLJ, et al. Implicación familiar en los cuidados del paciente crítico. Enferm Intensiva. 2003;14:96-108.

11. Garrouste-Orgeas M, Willems V, Timsit JF, Diaw F, Brochon $\mathrm{S}$, Vesin A, et al. Opinions of families, staff, and patients about family participation in care in intensive care units. J Cri Care. 2010;25:634-640.

12. Romero M, Delgado $P$, de la Cueva, Martínez MA, Lluch MA, Trujols J, et al. Level of satisfaction of critical care patients regarding the nursing care received: correlation with sociodemographic and clinical variables. Aust Cri Care. 2018;32:486-493. 
13. Fumagalli S, Boncinelli L, Lo Nostro A, Valoti P, Baldereschi G, Di Bari M, et al. Reduced cardiocirculatory complications with unrestrictive visiting policy in an intensive care unit results from a pilot, randomized trial. Circulation. 2006;113:946-952.

14. Adrieno G, Schirmer M, D'Aroz D, Caroline A, Machado MC, et al. Effect of a 24-h extended visiting policy on delirium in critically ill patients. Intensive Care Med. 2018;44:968-970.

15. Rosa R, Tonietto T, da Silva D, Gutierres F, Ascoli A, Madeira L. ICU Visits Study Group Investigators. Effectiveness and safety of an extended ICU visitation model for delirium prevention: a before and after study. Crit Care Med. 2017;45:1660-1667.

16. Gómez-Carretero P, Monsalve V, Soriano JF, de Andrés J. Alteraciones emocionales y necesidades psicológicas de pacientes en una Unidad de Cuidados Intensivos. Med Intensiva. 2007;31:318-325.

17. Wade DF, Moon Z, Windgassen SS, Harrison AM, Morris AM, Weinman JA. Non-pharmacological interventions to reduce ICUrelated psychological distress: a systematic review. Minerva Anestesiol. 2016;82:465-478.

18. Wolters A, Bouw M, Vogelaar, Tjan D, van Zanten A, van der Steen M. The postintensive care syndrome of survivors of critical illness and their families. J Clin Nurs. 2015;24:876-879.

19. Holanda MS, Marina N, Ots E, Lanza JM, Ruiz A, García A, et al., para el Grupo HU-CIC. Satisfacción en la Unidad de Cuidados Intensivos (UCI): la opinión del paciente como piedra angular. Med Intensiva. 2017;41:78-85.

20. Harvey M, Ninos N, Adler D, Goodnough-Hanneman S, Kaye W, Nikas D. Results of concensus conference on fostering more human critical care: creating a healing enviroment. AACN. 1993;4:484-507.

21. Zaforteza C, Sánchez C, Lastra P. Análisis de la literatura sobre los familiares del paciente crítico: es necesario desarrollar investigación en cuidados efectivos. Enferm Intensiva. 2008;19:61-70.
22. Rodríguez MC, Rodríguez F, Roncero A, Morgado MI, Theodor J, Flores LJ, et al. Implicación familiar en los cuidados del paciente crítico. Enferm Intensiva. 2003;14:96-108.

23. García A, Heras G, Serrano A. Revisión narrativa sobre humanización en cuidados intensivos pediátricos: ¿dónde estamos? Med Intensiva. 2019;43:290-298.

24. Jover C, Romero M, Delgado P, de la Cueva L, Solà N, Acosta $B$, et al. Percepción de las enfermeras de $\mathrm{UCl}$ en relación al cuidado satisfactorio: convergencias y divergencias con la percepción del paciente crítico. Enferm Intensiva. 2015;26:3-14.

25. Luiz FF, Aquino RC, da Costa ME. Humanization in the Intensive Care: perception of family and healthcare professionals. Rev Bras Enferm. 2017;70:1040-1047.

26. Delgado P, Sola A, Mirabete I, Torrents R, Blasco M, Barrero $\mathrm{R}$, et al. Modificación de la práctica enfermera a través de la reflexión: una investigación-acción participativa. Enferm Intensiva. 2001;12:110-126.

27. Haque OS, Waytz A. Dehumanization in medicine: causes, solutions, and functions. Perspect Psychol Sci. 2012;7:176-186.

\author{
Correspondencia: \\ Dr. Ricardo Martínez Zubieta \\ Unidad de Terapia Intensiva, \\ Hospital Español de México. \\ Avenida Ejército Nacional Núm. 613, Piso 9, \\ Col. Granada, CP 11520, \\ Alcaldía Miguel Hidalgo, Ciudad de México. \\ E-mail: ricardomz85@prodigy.net.mx
}

